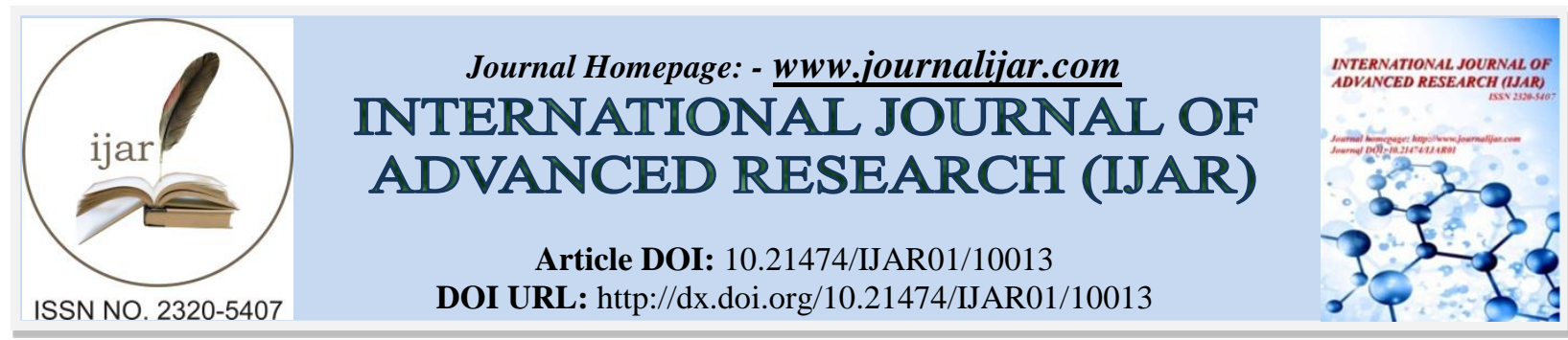

RESEARCH ARTICLE

\title{
STUDY OF SIX MINUTE WALK TEST IN PATIENT OF METABOLIC SYNDROME.
}

Tulika Kumari $^{1}$, Shyam Chand Choudhary ${ }^{1}$, Kauser Usman ${ }^{1}$, K.K. Sawlani ${ }^{1}$, Avinash Agrawal $^{2}$, M.L. Patel ${ }^{1}$, D. Himanshu ${ }^{1}$, K.K. Gupta ${ }^{1}$ and Ajay Verma ${ }^{3}$.

1. Department of Internal Medicine, King Georg's Medical University, Lucknow, Uttar Pradesh, India.

2. Department of Critical Care Unit, King Georg's Medical University, Lucknow, Uttar Pradesh, India.

3. Department of Pulmonary Medicine, King Georg's Medical University, Lucknow, Uttar Pradesh, India.

\section{Manuscript Info}

\section{Manuscript History}

Received: 08 September 2019

Final Accepted: 10 October 2019

Published: November 2019

Key words:-

Metabolic Syndrome, Six-Minute walk test, Non communicable disease.

\section{Abstract}

Introduction: South Asian countries are undergoing an epidemiological transition in the urban and sub urban area which is characterized by a decrease of infectious diseases and an increase in chronic non -infectious ones. This increase is largely due to a marked change in lifestyle (sedentary lifestyle, obesity, and stress of urbanization and labour), including changes in food consumption patterns. Although the cardiopulmonary exercise test remains the goldstandard method of assessing the individual's metabolic, cardiorespiratory fitness and maximal capacity for exercise, the high-intensity nature of exhaustive stress tests may be inappropriate for certain low-fit or patient groups. Therefore, sub maximal exercise testing may provide a safe, practical means of evaluating functional status, monitoring treatment effectiveness and establishing prognosis. Material and Methods: A cross sectional prevalence study was done in department of Medicine, KGMU, Lucknow on 100 patients of the age between 2565 years who fulfilled the IDF criteria for Metabolic Syndrome to study 6 minute walk test in metabolic syndrome. Result: Maximum number of patients were in 31-40 years of age group (45\%) followed by those aged 410 year $(26 \%)$, <30 years $(15 \%),>50$ years $(14 \%)$. Mean age of patients was $39.59 \pm 8.67$ year.Distance covered by subjects of control group $(405.65 \pm 12.73 \mathrm{~m})$ was found to be statistically significantly higher than that of metabolic syndrome subjects $(298.36 \pm 27.02 \mathrm{~m})$ during the 6 Min Walking Distance test.\% Predicted 6 min walking distance of control group $(86.56 \pm 2.18 \%)$ was found to be significantly higher than that of metabolic syndrome subjects (79.13 $\pm 0.64 \%)$.Difference in other hemodynamic variables (oxygen saturation respiratory rate, and pulse rate at rest $(\mathrm{PR}-\mathrm{R})$ and pulse rate during walking PR-W) among subjects of metabolic syndrome subjects and control were not found to be statistically significant.

Conclusion:Patients with Metabolic Syndrome showed reduced maximal exercise capacity as assessed by 6MWT as compared to healthy control. However, no significant correlation was found between Metabolic Syndrome patients with respect to change in HR, RR, Spo2 during 6MWT. 


\section{Introduction:-}

South Asia is home to one-quarter of the world's population. ${ }^{1}$ It is undergoing an epidemiological transition in the urban and sub urban area which is characterized by a decrease of infectious diseases and an increase in chronic non infectious ones. ${ }^{1}$ This increase is largely due to a marked change in lifestyle, including changes in food consumption patterns. ${ }^{1}$ Among non-infectious disease, Metabolic Syndrome is one of the most important one. It is defined as a constellation of an interconnected physiological, biochemical, clinical, and metabolic factors that directly increases the risk of atherosclerotic cardiovascular disease, T2DM. ${ }^{2}$ In a number of recent studies, it was reported that among the changes in pulmonary function, deterioration in pulmonary function is related to hypertension, type 2 diabetes, low-density lipoprotein cholesterol, overall obesity, abdominal obesity and insulin resistance. ${ }^{4}$ Among the above listed factors, hypertension, diabetes, and abdominal obesity are included as diagnostic criteria for Metabolic Syndrome. ${ }^{5}$ Assessment of functional capacity and exercise tolerance is commonly examined in obese subjects by cardiopulmonary exercise test with measurement of respiratory gas exchange, which requires sophisticated equipment and specially trained personnel. . Although the cardiopulmonary exercise test remains the gold-standard method of assessing the individual's metabolic, cardio-respiratory fitness and maximal capacity for exercise, the high-intensity nature of exhaustive stress tests may be inappropriate for certain low-fit or patient groups. Recently, the development of field tests, such as walking tests, permits to provide simply the measurement of functional exercise capacities of healthy or unfit subjects. The six-minute walking test (6MWT) has emerged as a common approach, and recent normative data have extended its application. ${ }^{6}$

\section{Material and Method:- Subject}

A cross sectional prevalence study was done in department of Internal Medicine, KGMU, lucknow on 100 patients of the age between 25-65 years who fulfilled the IDF criteria for Metabolic Syndrome to evaluate 6-Minute Walk Test in Metabolic Syndrome.It was done over a period of 1 year from Sep 2016 to Aug 2017 and patients were enrolled from OPD and those admitted in indoor wards. Patients with cardiac, endocrine, pulmonary, orthopedic, or neurogenic condition and any systemic or surgical illness were excluded from the study.

Study protocol

The subjects were informed about the study through information sheets, and written consent was obtained from all subjects. The study was approved by Research and Ethical Committee of the institute. Testing was performed in the hospital where a rapid, appropriate response to an emergency was possible, and physician was also available on call whenever any emergency arises. A detailed clinical history and physical examination carried out for every subject. History of presence of risk factors, like smoking, hypertension, dyslipidaemia, diabetes mellitus and presence of any other chronic disease was inquired. The anthropometric characteristics, blood pressure, plasma glucose, and lipid levels, were measured. Metabolic syndrome was defined clinically, based on IDF criteria which include waist circumference $>90 \mathrm{~cm}$ in males, $>80 \mathrm{~cm}$ in females and two or more of the following, a high triglyceride level $(>150 \mathrm{mg} / \mathrm{dl})$ or on specific medication, a low high-density lipoprotein-cholesterol (HDL-C) level ( $<40 \mathrm{mg} / \mathrm{dl}$ for men and $<50 \mathrm{mg} / \mathrm{dl}$ for women) or on specific medication, high blood pressure $(\geq 130 / 85 \mathrm{~mm} \mathrm{Hg})$ or on specific medication, and a high fasting plasma glucose concentration $(>100 \mathrm{mg} / \mathrm{dl})$ or on specific medication or previously diagnosed type 2 DM / Impaired fasting glucose/ impaired glucose tolerance. Subsequently, 6-Minute Walk Test was done.

\section{Minute Walk Test}

There are several modalities available for the objective evaluation of functional exercise capacity. Some provide a very complete assessment of all systems involved in exercise performance (high tech), whereas others provide basic information but are low tech and are simpler to perform. The modality used should be chosen based on the clinical question to be addressed and on available resources. The most popular clinical exercise tests is 6MWT. LocationThe 6MWT was performed indoors, along a long, flat, straight, enclosed corridor with a hard surface that was seldom travelled. The walking course was $30 \mathrm{~m}$ in length. A 100-ft hallway was, therefore, chosen. The length of the corridor was marked every $3 \mathrm{~m}$. The turnaround points was marked with a cone (such as an orange traffic cone). A starting line, which marks the beginning and end of each 60-m lap, was marked on the floor using brightly coloured tape. Patients were instructed as follows: "The object of this test is to walk as far as possible for 6 minutes. You will walk back and forth in this hallway. Six minutes is a long time to walk, so you will be exerting yourself. You will probably get out of breath or become exhausted. You are permitted to slow down, to stop, and to rest as necessary. 
You may lean against the wall while resting, but resume walking as soon as you are able. You will be walking back and forth around the cones. You should pivot briskly around the cones and continue back the other way without hesitation. Now I'm going to show you. Please watch the way I turn without hesitation." Demonstration was given by walking one lap myself. "Are you ready to do that? I am going to use this counter to keep track of the number of laps you complete. I will click it each time you turn around at this starting line. Remember that the object is to walk AS FAR AS POSSIBLE for 6 minutes, but don't run or jog. Start now, or whenever you are ready."

1. Patients were positioned at the starting line. Timer was started as soon as the patient started to walk.

2. Each time the participant returned to the starting line, lap counter was clicked once.

3. Post-test: Post walk Borg dyspnoea and fatigue levels was recorded and was asked: "What, if anything, kept you from walking farther?"

4. Using pulse oximeter, $\mathrm{SpO} 2$ and pulse rate was measured.

5. Number of laps from the counter (or tick marks on the worksheet) was measured. Additional distance covered was measured (the number of meters in the final partial lap) using the markers on the wall as distance guides. Total distance walked was calculated, rounding to the nearest meter, and was recorded it on the worksheet.

6. Patients were congratulated on good effort and offered a drink of water.

\section{Serum sampling and biochemical analysis}

Blood samples were obtained following 12 hours of fasting were immediately centrifuged ( $3000 \mathrm{rpm})$ for 10 minute; the sera were separated and frozen at $-8{ }^{\circ} \mathrm{C}$ until analysis. Fasting blood glucose (FBG), total cholesterol, triglycerides (TG), and high density lipoprotein cholesterol (HDL-C) levels were determined by enzymatic method using commercial available diagnostic kit on fully automated biochemical analyzer. Low density lipoproteins cholesterol (LDL-C) was determined by using Friedewald formula (Friendewald et al., 1972).

\section{Statistical analysis:-}

The statistical analysis was done using SPSS (Statistical package for social science) Version 15.0 statistical analysis software. The values were represented in No (\%), Mean \pm SD. Student's t-test was used while assessing spirometry data. $\mathrm{P}<0.05$ was considered statistically significant.

\section{Result:-}

A total of 100 patients of Met-S fulfilling the inclusion criteria of the study were enrolled as cases (male-70, female30 , mean age-39.59 \pm 8.67 ) and classified as group I while 100 age gender matched controls (normal healthy subjects, male- 66 , female-34, mean age-42.81 \pm 9.45 ) were also included in the study and classified as group II.

On comparing the hematological and biochemical variables, statistically significant difference among patients of group I and group II was observed in SGPT, S ALP and S. Albumin levels only. SGPT level of subjects of group I $(90.18 \pm 232.58 \mathrm{U} / \mathrm{l})$ was found to be significantly higher than that of group II( $42.32 \pm 24.79 \mathrm{U} / \mathrm{L})$. In the same way ALP level of subjects of group I $(232.48 \pm 84.50 \mathrm{IU} / \mathrm{L})$ was found to be significantly higher than that of group II $(191.89 \pm 130.04 \mathrm{IU} / \mathrm{L})$ and mean serum albumin levels of group II (3.31 \pm 0.61$)$ were found to be significantly higher than that of group I $(3.07 \pm 0.63)$ (Table 1$)$.

Table 1:-Comparison of biochemical/hematological variables between two groups

\begin{tabular}{|l|l|l|l|l|}
\hline Variables & Group I & Group II & \multicolumn{2}{l|}{ Student 't' test } \\
& & N-100 & \multicolumn{2}{|l|}{} \\
\cline { 2 - 5 } & Mean \pm SD & Mean \pm SD & 't' & 'p' \\
\hline Hb (g/100 ml) & $11.92 \pm 1.28$ & $11.89 \pm 1.27$ & 0.199 & 0.842 \\
\hline TLC (cells/cumm) & $8255.40 \pm 2806.83$ & $8125.9 \pm 2800.21$ & 0.327 & 0.744 \\
\hline DLC (Neutrophile \%) & $70.69 \pm 7.88$ & $70.74 \pm 8.14$ & -0.044 & 0.965 \\
\hline DLC (Lymphocyte \%) & $33.09 \pm 6.75$ & $33.47 \pm 6.52$ & -0.405 & 0.686 \\
\hline Platelet (Lacs) & $1.95 \pm 0.58$ & $9.08 \pm 45.34$ & -1.574 & 0.117 \\
\hline S. bilirubin (mg\%) & $0.85 \pm 0.47$ & $0.87 \pm 1.05$ & -0.207 & 0.836 \\
\hline SGOT (U/L) & $75.31 \pm 175.44$ & $42.55 \pm 31.32$ & -1.836 & 0.068 \\
\hline SGPT (U/L) & $90.18 \pm 232.58$ & $42.32 \pm 24.79$ & 2.046 & 0.042 \\
\hline ALP (IU/L) & $232.48 \pm 84.50$ & $191.89 \pm 130.04$ & 2.597 & 0.010 \\
\hline
\end{tabular}




\begin{tabular}{|l|l|l|l|l|}
\hline S. Protein $(\mathrm{g} / \mathrm{dl})$ & $6.17 \pm 0.52$ & $6.27 \pm 1.07$ & 0.859 & 0.392 \\
\hline S. Albumin $(\mathrm{g} / 100 \mathrm{ml})$ & $3.07 \pm 0.63$ & $3.31 \pm 0.61$ & -2.699 & 0.008 \\
\hline S. Uric $\mathrm{acid}(\mathrm{mg} / \mathrm{dl})$ & $5.547 \pm 2.02$ & $5.70 \pm 2.18$ & 0.548 & 0.584 \\
\hline S. Urea $(\mathrm{mg} / \mathrm{dl})$ & $35.94 \pm 8.08$ & $35.48 \pm 8.27$ & 0.398 & 0.691 \\
\hline S. Creatinine & $1.21 \pm 0.35$ & $1.20 \pm 0.35$ & 0.234 & 0.815 \\
\hline
\end{tabular}

Among group I, proportion of subjects with obesity, hypertension, raised fasting blood sugar level, increased triglyceride level, and low HDL level were $100 \%, 79 \%, 100 \%, 88 \%, 48 \%$ respectively. In the same way, among group II proportion of subjects with obesity, hypertension, raised fasting blood sugar level, increased triglyceride level, and low HDL level were $21 \%, 9 \%, 1 \%, 1,0 \%$ respectively. This difference among different variables of MetS between two groups was found to be statistically significant $(\mathrm{p}<0.001)$ (Table 2$)$.

Table 2:-Comparison of different variable of Met S between group I, and II

\begin{tabular}{|l|l|l|l|l|}
\hline & $\begin{array}{l}\text { Group I } \\
(\mathbf{N}-\mathbf{1 0 0})\end{array}$ & $\begin{array}{l}\text { Group II } \\
(\mathbf{N}-100)\end{array}$ & $\chi^{\mathbf{2}}$ & 'P' value \\
\hline Obese & 100 & 21 & $130.579(\mathrm{df}=1)$ & $<0.001$ \\
\hline Hypertensive & 79 & 9 & $99.432(\mathrm{df}=1)$ & $<0.001$ \\
\hline $\begin{array}{l}\text { Fasting Blood Sugar level } \\
(>100 \mathrm{mg} / \mathrm{dl})\end{array}$ & 100 & 1 & $196.040(\mathrm{df}=1)$ & $<0.001$ \\
\hline $\begin{array}{l}\text { Hypertriglyceredemia } \\
(>150 \mathrm{mg} / \mathrm{dl})\end{array}$ & 88 & 1 & $153.234(\mathrm{df}=1)$ & $<0.001$ \\
\hline
\end{tabular}

Distance covered by subjects of group II $(405.65 \pm 12.73 \mathrm{~m})$ was found to be statistically significantly higher than that of group I $(298.36 \pm 27.02 \mathrm{~m})$ during the 6 Min Walking Distance test.\% Predicted 6 min walking distance of group II $(86.56 \pm 2.18 \%)$ was found to be significantly higher than that of group I $(79.13 \pm 0.64 \%)$.Difference in other hemodynamic variables (oxygen saturation, respiratory rate, and pulse rate at rest (PR-R) and pulse rate during walking PR-W) among subjects of group I and group II were not found to be statistically significant (table 3).

Table 3:-Relationship between Six Minute Walk test variables and Metabolic Syndrome

\begin{tabular}{|l|l|l|l|l|}
\hline \multirow{2}{*}{ Variables } & Group I & Group II & \multicolumn{2}{l|}{ Student 't' test } \\
\cline { 2 - 5 } & Mean \pm SD & Mean \pm SD & 't' & 'p' \\
\hline 6MWD & $298.36 \pm 27.02$ & $405.65 \pm 12.73$ & -35.471 & $<0.001$ \\
\hline \% Predicted 6MWD & $79.13 \pm 0.64$ & $86.56 \pm 2.18$ & -32.678 & $<0.001$ \\
\hline SPo2 & $97.23 \pm 1.20$ & $97.33 \pm 1.12$ & -0.608 & 0.544 \\
\hline RR & $16.83 \pm 0.69$ & $16.81 \pm 0.69$ & 0.210 & 0.834 \\
\hline PR-R & $77.19 \pm 1.70$ & $77.16 \pm 1.68$ & 0.111 & 0.912 \\
\hline PR-W & $108.55 \pm 1.89$ & $108.42 \pm 1.99$ & 0.473 & 0.637 \\
\hline
\end{tabular}

\section{Discussion:-}

Patients with Metabolic Syndrome showed reduced maximal exercise capacity as assessed by 6MWT (table 3) as compared to normal control. However, no significant correlation was found between metabolic syndrome and nonMetabolic Syndrome patients with respect to change in HR, RR, Spo2 during 6MWT. LuporiniI et al did a similar cross sectional prospective study to analyze and compare the cardiopulmonary, metabolic, and perceptual responses during a cardiopulmonary exercise test (CPX) and a treadmill six-minute walking test (tread6MWT) in obese and eutrophic women. He found a significant correlation between decline in maximum exercise capacity as assessed by 6MWT and obese patients which is consistent with our result. ${ }^{7}$

\section{Conclusion:-}

Patients with Metabolic Syndrome showed reduced maximal exercise capacity as assessed by 6MWT as compared to healthy control. However, no significant correlation was found between Metabolic Syndrome patients with respect to change in HR, RR, Spo2 during 6MWT. 


\section{References:-}

1. Institute for Health Metrics and Evaluation. The Global Burden of Disease: Generating Evidence, Guiding Policy - South Asia Regional Edition. Seattle, WA: Institute for Health Metrics and Evaluation, University of Washington and Human Development Network, The World Bank; 2013.

2. Wang J, Ruotsalainen S, Moilanen L, Lepistö P, Laakso M, Kuusisto J. The metabolic syndrome predicts cardiovascular mortality: a 13-year follow-up study in elderly non-diabetic Finns.Eur Heart J;2007 Apr;28(7):857-64.

3. Young RP, Hopkins R, Eaton TE. Forced expiratory volume in one second: not just a lung function test but a marker of premature death from all causes. Eur Respir J. 2007 Oct;30(4):616-622.

4. Lin WY, Yao CA, Wang HC, Huang KC. Impaired lung function is associated with obesity and metabolic syndrome in adults. Obesity (Silver Spring) .2006;14:1654-1661.

5. International Diabetes Federation. The IDF consensus worldwide definition of the metabolic syndrome, 2005. www.idf.org

6. Rydén L1, Standl E, Bartnik M, Van den Berghe G, Betteridge J, de Boer MJ et al. Guidelines on diabetes, prediabetes and cardiovascular diseases: executive summary. The Task Force on Diabetes and Cardiovascular Diseases of the European Society of Cardiology (ESC) and of the European Association for the Study of Diabetes (EASD).Eur Heart J. 2007 Jan;28(1):88-136.

7. Luporini 1,Soraia P.Jürgensen,Viviane Castello-Simões, Aparecida M. Catai' Ross Arena et al. Prediction of Cardiorespiratory Fitness by the Six-Minute Step Test and Its Association with Muscle Strength and Power in Sedentary Obese and Lean Young Women: A Cross-Sectional Study. PLoS One. 2015; 10(12): e0145960. 